\title{
Orbifold Construction in Subfactors
}

\section{Feng Xu}

\author{
Mathematics Department, U.C. Berkeley, CA94720, USA
}

Received: 30 June 1992

\begin{abstract}
We use the lattice models to determine the obstructions to the flatness of the orbifold connections in some finite depth subfactors.
\end{abstract}

0. The motivation of the present work is the question raised in [1]. In [1], the author applied orbifold construction, first used in [2], to the subfactors coming from the Hecke algebra. The key notion is the flatness of the connection in [3]. A connection is an assignment of a complex number to cells, and flatness is a condition on the connection. A more detailed description is included in the appendix. To prove the flatness, one needs certain identities involving a large number of quantities determined by the connection. For $S U(N), N$ odd, and subfactors corresponding to vector representations of $S U(N)$, the flatness of the orbifolding subfactors can be derived from (See [1]):

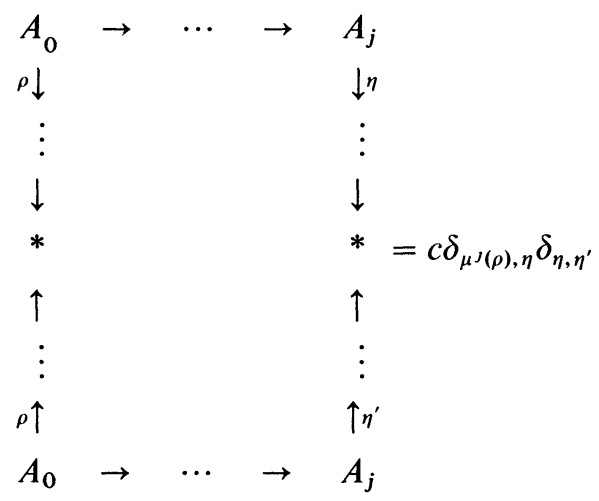

Here $c$ is a constant. (By using simple argument one can show $c=1$.) The symbol on the right-hand side is the notation for the connections, see $[1,2,3]$ or the appendix for the precise definitions. In [1], (1) is proved under certain assumptions by using the flatness of Jones projections. It seems to be hard to use this method 
without these assumptions. In this paper, we use integrable lattice models of [12], also used in subfactors setting in [4], to prove the following results:

(a): (1) holds for any $N$;

(b): When $N$ is even, the flatness holds iff $2 N \mid K$;

The case $N=2$ is proved in [2].

It turns out that the same method applies to the more general case.

Let $G$ be a connected, simply connected, compact simple Lie group with nontrival center, i.e. $G=S U(N), S O(2 N+1), S O(2 N), S P(2 N), E_{6}, E_{7}$. Let $Z$ be a non-trivial subgroup of the center $Z(G)$ of $G$. Let $\theta_{z}$ denote the set of fundamental weights of $G$ associated to $Z$. Let $M$ be the least natural number such that:

$$
1 / 2 M\left(\theta_{z}, \theta_{z}\right) \in \mathbb{Z} \quad \forall 0_{z} \in \theta_{Z} .
$$

Let $\phi$ be a finite dimensional representation of $G . K \in N$ is a fixed integer (level). In [4], a coupling system associated to $G_{K}$ Wess-Zumino-Witten model and field $\phi$ is constructed, denoted by $\left(g_{\phi}(K), h_{\phi}(K), B, \tau\right)$. Here $g_{\phi}(K)$ is the principal graph constructed out of the fusion graph of $\phi, h_{\phi}(K)$ is its dual.

If $K$ is such that:

$$
Z(0) \in g_{\phi}^{\text {even }} \cap h_{\phi}^{\text {even }} .
$$

One can apply the orbifold with respect to $Z$ to this coupling system as in [1] and [2].

Theorem. Let $K$ be as in (2). The connection of the orbifold subfactor is flat iff $M \mid K$.

The following examples are applications of the theorem:

e.g. 1: Let $G=S U(N), \phi$ the vector representation of $G . Z=Z_{t}$ is a subgroup of the center group $Z_{N}$. It is possible to do the orbifold construction if $t \mid K$; the theorem says the connection of the orbifold is flat iff $t \mid K$ when $t$ is odd and $2 t \mid K$ if $t$ is even. This answers one of the main questions in [1] completely.

e.g. 2: Let $G=S U(2), \phi$ the spin 1 representation. Then (2) says $K \in 2 \mathbb{Z}$ and (3) says $K \in 4 \mathbb{Z}$.

e.g. 3: Let $G=S O(2 N+1)(N \geqq 2), \phi$ the vector representation of $G$. Then (2) and (3) says $K \in 2 \mathbb{Z}$.

Remark. [a]: A Chern-Simon gauge theory based on $G / Z$ exists at level $K$ iff (2) holds, see (8). This is actually how (3) comes from.

[b]: The condition (3) is the first necessary condition when one is using the orbifold in a string algebra construction as in [1]. The reason is that one needs the following strings:

$$
O \stackrel{\alpha}{\rightarrow} \cdots \rightarrow \cdots+\stackrel{\beta}{\leftarrow} Z(0) .
$$

Here the length of $\alpha$ is equal to that of $\beta$. Since $g, h$ are bipartiegraphs, $Z(0)$ must have the same color as 0 for any $z \in Z$. (See [3] for the definitions).

The content of the paper is as follows: In Sect. 1, we describe some of the results of $[4,12$ and 7$]$ which we will need to fix the notations. In Sect. 2, we apply the orbifold method [1] and [2] to the coupling system described in Sect. 1. Lemma 2.17 answered the motivating question in the introduction. Lemma 2.21 deals with the $S U(N)$ case when $N$ is even. We also include an appendix on part of the general theory of subfactors for the convenience of the reader. 


\section{Integrable Lattice Models and Coupling System}

The material of this is contained in [4, 5 and 7]. Let's begin by assigning numbers to the following pictures:

$$
\begin{aligned}
& B_{j l}\left[\begin{array}{ll}
m & n \\
i & k
\end{array}\right](+)={ }_{m p}\left[\begin{array}{ll}
r & s \\
i & k
\end{array}\right]=\underbrace{\mathrm{k}}_{\mathrm{m}},
\end{aligned}
$$

These $B, F$ (for simplicity, we omit the labels or assume $N_{i j}^{k}=1,0$ for the time being) are the so-called braiding and fusion matrices respectively in conformal field theory. There are special fusion matrices:

$$
F_{j k}^{i}=\overbrace{i}^{\mathrm{k}}\left(F_{j k}^{i}\right)^{r}=\underbrace{\mathrm{k}}_{i}
$$

They are nonzero numbers. These matrices satisfy the Yang-Baxter equation and braiding-fusion relations as described by the following pictures:

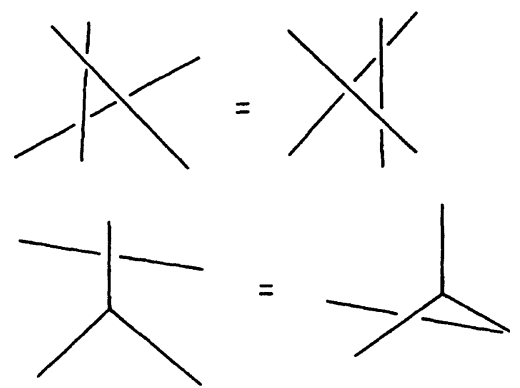

More precisely these pictures should be framed. It's conventional to choose the blackboard framing, i.e. the normal vector points out to the reader. The change of framing is given by:

$$
\emptyset_{0}=\exp \left( \pm 2 \pi i h_{i}\right) \quad i
$$

Here + or - depends on the convention, and $h_{i}$ is the conformal dimension. There's another ingredient, so-called $S$-matrix, or the modular transformation matrix in conformal field theory besides $B$ and $F$. The matrix elements of $S$ is 
denoted by $S_{i j}$ in the following. Here $i \in I, j \in I$ (see (b) for the definition of $I$ ). Let $\chi_{i}=S_{0 i} / S_{00}$. It's proved in [5 and 7] that one can make a "good" gauge choice such that:

$$
\begin{aligned}
& { }_{i} k f_{i}=\left(\frac{\chi_{k}}{\chi_{i} \chi_{j}}\right)^{1 / 2} \\
& \overbrace{\mathrm{k}}^{\mathrm{i}}=\left(\frac{\chi_{k} \chi_{j}}{\chi_{i}}\right)^{1 / 2} \\
& h_{0}^{i} \hat{k}^{\mathrm{k}} \boldsymbol{f}^{\mathrm{k}}=\left(\chi_{i} \chi_{j} \chi_{k}\right)^{1 / 4} \\
& B_{j l}\left[\begin{array}{ll}
m & n \\
i & k
\end{array}\right](+)=\left[\begin{array}{c}
1 \\
\left(\chi_{i} \chi_{m} \chi_{n} \chi_{k}\right)^{1 / 2}
\end{array}\right.
\end{aligned}
$$

Now let's turn to the integrable lattice models. Integrable lattice models are devised be Witten to given a lattice field theory approach to Chern-Simons gauge theory. To explain the rules, we have to explain the following notions:

(a) One choose a plane where all trivalent graphs (in a generic position) in $S^{3}$ are projected onto. On this plane where one must choose a time direction. The projection must be generic in the sense that the equal time slice will intersect the projected graphs with a finite number of points.

(b) Painting the projected graphs with colors. In RCFT there's a finite index set $I$. There's a finite dimensional algebra $A$, called Verlinde's fusion algebra, with basis $(i)_{i \in I}$ and multiplications. $i \times j=\sum_{k} N_{i j}^{k}$. Hence $N_{i j}^{k} \in \mathbb{Z}_{\geqq 0}$; they're called fusion coefficients. The algebra $A$ is also endowed with an involution $\tau: A \rightarrow A$ and there is a distinguished element $0 \in A$ (so-called vacuum) such that $\tau(0)=0$ and $N_{i i}^{0}=1$, $N_{0 i}^{j}=\delta_{i j}$. The coupling space, denoted by $V_{i j}^{k}$, has dimension $N_{i j}^{k}$. A choice of basis in this coupling space is called a gauge choice. We always assume we make a "good" gauge choice as in [5 and 7]. A basis of the coupling space is denoted by $\varepsilon_{1}, \ldots, \varepsilon_{a}$ with $a=N_{i j}^{k}$. They are sometimes called spin variables. The index set is called a set of colors. Colors $(i, j ; k)$ are called admissible if $N_{i j}^{k} \geqq 1$.

( ${ }^{t}$ ) Critical points. They are 4 kinds of critical points as seen from the following pictures:
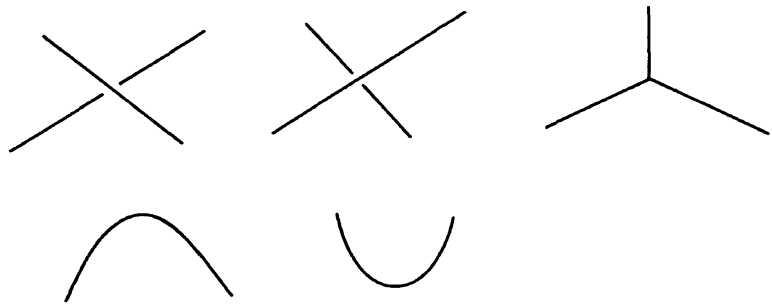
We use the following example to explain the above rules:

The diagram consists of two concentric circles.

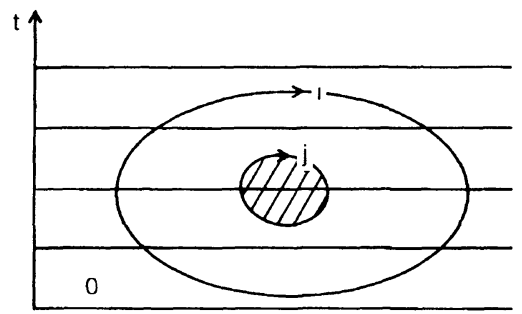

One chooses a time direction and equal time slices $t=t_{0}, t_{1}, t_{2}, t_{3}, t_{4}$ such that between $t_{i}$ and $t_{i+1}$ there's only one critical point. Each line is endowed with an element of the index set $I$, and so is each region separated by the lines. The line separating two regions is endowed with a spin variable. For example,

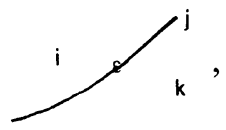

where $\varepsilon \in V_{i j}^{k}$. So it's necessary to have $(i, j ; k)$ admissible, i.e., $N_{i j}^{k} \geqq 1$. The spin variable is always omitted in the following for simplicity. One should bear in mind that it is possible to include them at each stage. After painting, one assigns numbers of (1.1), (1.2) and (1.3) to the critical points of (1.15), take their produce and sum over all the closed regions to get the partition function of the projected graph. It's proved in [5] that it's a topological invariant by showing its invariance under Redemster moves of type two and three. (So more precisely, it's an invariant of framed graphs.)

Let's use the graph (1.16) to illustrate that its value is topologically invariant. The reader may find that in the case of topological field theory coming from the quantum at roots of unity, this fact is equivalent to the truncated Weyl character formula for the tensor product of two restricted representations $i$ and $j$.

Example: One finds in (1.16):

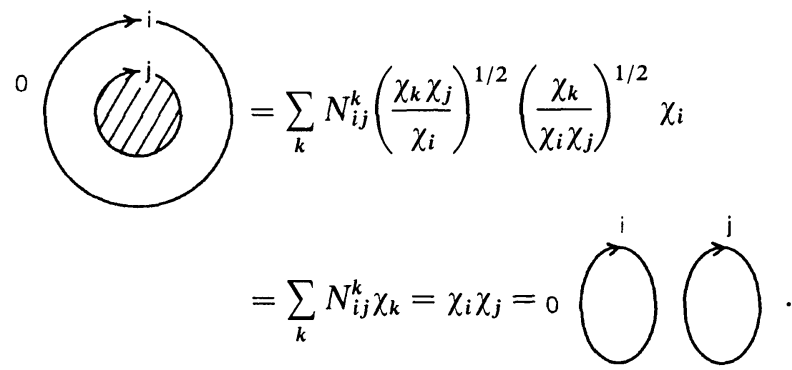

Finally, we describe the coupling system following [4], referring to the definition in [3]. See also the appendix.

In [4], a coupling system is constructed for an RCFT and a primary field $\phi$. One first makes a graph by taking $2 N$ vertices if $N$ is the number of primary fields in the theory, and label them by $\phi_{i}$ and $\phi_{j}^{\prime}$, where $i, j$ runs from 1 to $N$. Next we 
draw $N_{\phi i}^{j}$ edges from $\phi_{i}$ to $\phi_{j}^{\prime}$. Let $g$ be the connected component of the graph containing the distingushed element 0 . Let $\star_{g}=0$. Also let $h$ be the connected component containing $0^{\prime}$ and let $\star_{h}=0^{\prime}$. We see that $g$ is the graph obtained by alternatively fusing with $\phi$ and its dual $\hat{\phi}$, and $h$ is obtained by the same process, starting however with $\hat{\phi}$. The eigenvalues for the Perron-Frobenius eigenvector are given by $\left\|\Lambda_{g}\right\|=\left\|\Lambda_{h}\right\|=S_{0 \phi} / S_{00}$. The contragradient map is defined by $\tau\left(\phi_{i}\right)=\hat{\phi}_{i}$. The definition of the connection $W$ for the coupling system is a little bit more involved. Roughly speaking, it's suitably normalized braiding matrices mentioned earlier. We refer to [4] for details to. $(g, h, W, \tau)$ gives a coupling system in the sense of Oceanu. This is also proved in [4]. We give some examples following this construction.

Example 1. Takes $S O(2 n)_{2}$ Wess-Zumino-Witten model and the primary field corresponding to the vector representation of $S O(2 n)$. The $g$ is given by $D_{n}^{(1)}$ and $h=g$. This gives us a subfactor of index 4 .

Example 2. Take $S O(2 p+1)_{2} \mathrm{WZW}$ model, let $p \geqq 3$. The fundamental weights of chiral $\hat{S} O(2 p+1)$ (stands for the affine algebra associated to $S O(2 p+1)$ ) are given by $\omega_{0}, \omega_{1} \ldots \omega_{p}$ : here $\omega_{p}$ corresponds to the spin representation. Any dominant weight is given by $\lambda_{0} \omega_{0}+\lambda_{1} \omega_{1}+\ldots+\lambda_{p} \omega_{p}$, where $\lambda_{i} \geqq 0$ are integers. If the level is 2 , then $\lambda_{0}+\lambda_{1}+2 \lambda_{2}+2 \lambda_{3}+\ldots+2 \lambda_{p-1}+\lambda_{p}=2$. There are $p+2$ primary fields of level 2 , given by: $2 \omega_{0}, \omega_{0}+\omega_{1}, \omega_{2}, \ldots \omega_{p-1}$, $\omega_{0}+\omega_{p}, \omega_{p}=\omega_{1}, 2 \omega_{1}, 2 \omega_{p}$. Let $\phi$ be the primary field associated to $\omega_{0}+\omega_{p}$, the spin field. According to the methods of [9], the fusion rules are calculated: $\left(\omega_{p}+\omega_{0}\right) \times\left(\omega_{p}+\omega_{0}\right)=\left(\omega_{1}+\omega_{0}\right)+\omega_{2}+\ldots+\omega_{p-1}+2 \omega_{p}+2 \omega_{0}$.

$$
\begin{aligned}
\left(\omega_{p}+\omega_{0}\right) \times 2 \omega_{p} & =\left(\omega_{p}+\omega_{0}\right) \times \omega_{2}=\ldots \\
& =\left(\omega_{p}+\omega_{0}\right) \times \omega_{p-1} \\
& =\left(\omega_{p}+\omega_{0}\right) \times\left(\omega_{1}+\omega_{0}\right) \\
& =\left(\omega_{p}+\omega_{0}\right)+\left(\omega_{1}+\omega_{p}\right), \\
\left(\omega_{p}+\omega_{0}\right) \times 2 \omega_{0} & =\omega_{0}+\omega_{p}, \\
\left(\omega_{1}+\omega_{p}\right) \times\left(\omega_{0}+\omega_{p}\right) & =2 \omega_{1}+2 \omega_{2}+\ldots+\omega_{p-1}=2 \omega_{p}, \\
2 \omega_{1} \times\left(\omega_{0}+\omega_{p}\right) & =\omega_{1}+\omega_{p} .
\end{aligned}
$$

These fusion rules are sufficient to determine the Bratelli diagrams of certain inclusions of finite dimensional algebras, it is exactly the same as in [10], p. 12, with $N=2 p+1$. Hence the metaplectic invariants of links with $N=2 p+1$ an odd prime is the same as link invariants coming from Chern-Simons gauge theory with gauge group $S O(2 p+1)$, level 2 and with the link colored by spin representation of $S O(2 p+1)$.

\section{Orbifold Construction in Subfactors}

Let $G$ be a compact, connected, simply connected and simple Lie group. Let $Z$ be a nontrival subgroup of the center $Z(G)$ of $G$. The following is a list of all the 
possible cases: (with $Z(G)$ nontrival):

$$
\begin{gathered}
A: S U(N),(N \geqq 2), Z(S U(N))=Z_{N}, \quad Z=Z_{t} \leqq Z_{N}, t \mid N, \\
B: S O(2 n+1),(n \geqq 2), Z(S O(2 n+1))=Z_{2}, \quad Z=Z_{2}, \\
C: S P(2 n)(n \geqq 2), Z(S P(2 n))=Z_{2}, \quad Z=Z_{2}, \\
D: S O(2 n)(n \geqq 2), Z(S O(2 n))=Z_{2} \times Z_{2} \text { or } Z_{4}, \quad Z=Z_{2}, \quad Z_{4} \text { or } Z_{2} \times Z_{2}, \\
E_{6}, Z\left(E_{6}\right)=Z_{3}, \quad Z=Z_{3}, \\
E_{7}, Z\left(E_{7}\right)=Z_{2}, \quad Z=Z_{2},
\end{gathered}
$$

Orbifold construction in subfactors in first used in [2] to prove the nonexistence of $D_{\text {odd }}$ as a principal graph and realizes $D_{\text {even }}$ as the principal graph. We refer the detail to [1 and 2]. Let $K$ be a natural number, called level. Let $\phi$ be an irreducible representation of $G$. Let $(g, h, w, \tau)$ be the coupling system associated to $G_{k}$ WZW model and primary field $\phi$. Our intention is to apply the orbifold method with respect to $Z$ (a subgroup of $Z(G)$ ) to $(g, h, w, \tau)$. We use a slightly different notation for connection (for the definition, see appendix) from that of [1 and 2]. i.e.,

$$
\begin{array}{lll}
a & \rightarrow & b \\
\downarrow & \downarrow=\frac{a \mid b}{c \mid d} \\
c & \rightarrow & d
\end{array}
$$

The arrows on RHS of the (2.7) will be determined from the positions of $a, b, c$ and $d$ on $g$ and $h$, the same as in [4]. The following identities will be crucial for our purposes: (See [9])

$$
\begin{aligned}
& N_{\mu z(i)}^{z(\alpha)}=N_{\mu \gamma}^{\alpha}, \\
& N_{z(\mu)_{i}}^{\alpha}=N_{\mu_{i}}^{z(\alpha)}, \\
& S_{0 z(\mu)}=S_{0_{\mu}}, \forall z \in Z .
\end{aligned}
$$

In particular, in (2.8), let $\mu=0$, we get:

$$
Z(0) \times \mu=Z(\mu) .
$$

Here $Z(\mu)$ means the action of the central elements $z$ on the weights $\mu . \mu, \gamma, \alpha$ are within the truncated Weyl chamber determined by the level $K$. To apply the orbifold constructions to the double string algebra construction. We need two conditions:

1. $K$ must be such that $Z(0) \in g^{\text {even }} \cap h^{\text {even }} \forall z \in Z$. The reason is explained in remark [b] of the introduction. (2.11)

2. The connection is invariant under the action of $Z$. (2.12).

To prove (2.12), let us notice the following simple fact: (It's also used in [7]).

Lemma 2.13. Let $g_{0}$ be a closed graph with the outer region colored by 0 and $g_{i}$ be the same graph but with the outer region colored by $i \in I$. Then the partition function of $g_{0}\left(\right.$ denoted by $\left.Z\left(g_{0}\right)\right)$ is equal to that of $g_{i}\left(\right.$ denoted by $\left.Z\left(g_{i}\right)\right)$.

Proof. One introduces a large circle colored by $i$ (the outer region of the circle is colored by 0 ) whose interior contains $g$. 
Then:

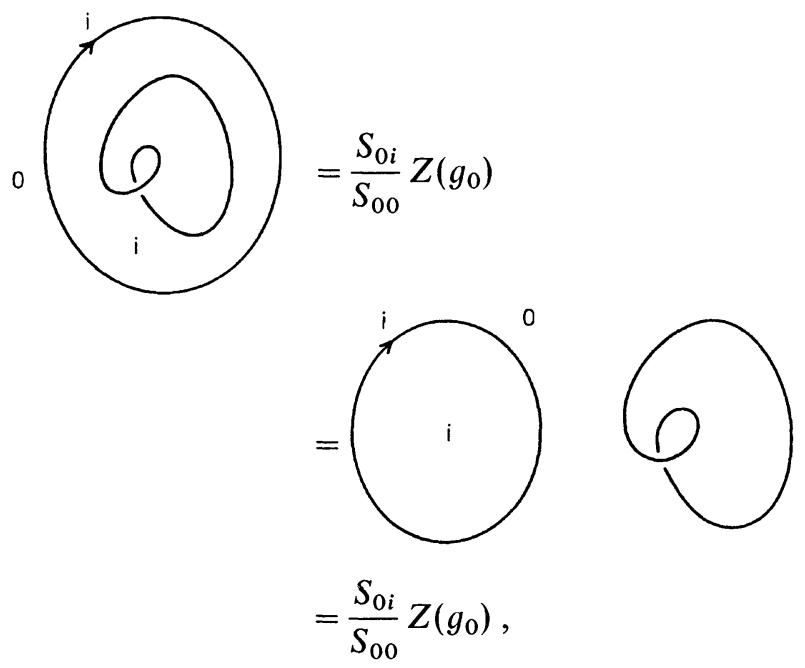

where the second identity follows from the topological invariance. Hence $Z\left(g_{i}\right)=Z\left(g_{0}\right)$. Apply Lemma 2.13 to the following picture with $i=z(0), z \in$ $Z, Z \leqq Z(G)$,

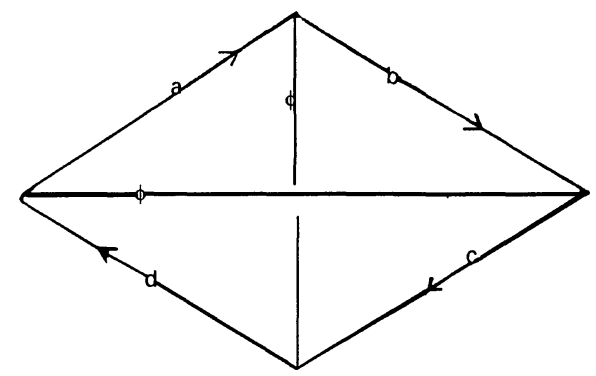

We get:
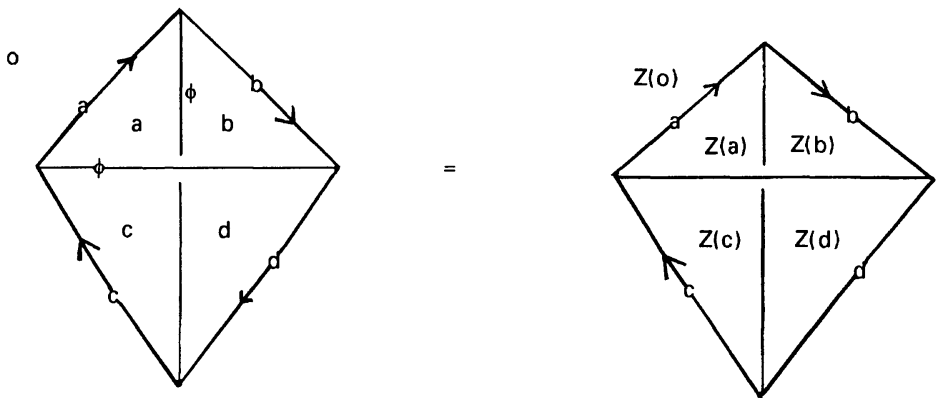

where we use the fact $Z(0) \times a=Z(a)$, from which one can assign only one color to the closed region in order to have admissible colors. See Sect. 1.1(c) for the definition of admissible colors. 
By (1.11):

$$
\begin{aligned}
& \begin{array}{ll}
a & b \\
\downarrow & \downarrow=\frac{1}{\left(\chi_{a} \chi_{d} \chi_{\phi}^{2}\right)^{1 / 2}}
\end{array} \\
& \begin{array}{ccc}
z(a) & \rightarrow & z(b) \\
\downarrow & \downarrow \\
z(c) & \rightarrow & z(d)
\end{array}=\frac{1}{\left(\chi_{z(a)} \chi_{z(d)} \chi_{\phi}^{2}\right)^{1 / 2}}
\end{aligned}
$$

From (2.14) and (2.15),

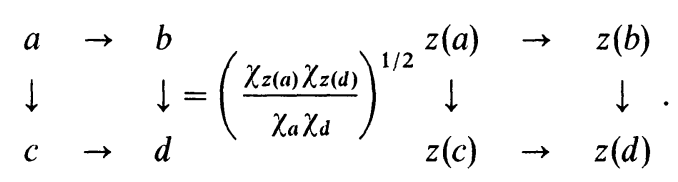

By (2.9), $\chi_{z(a)}=\chi_{a}$, thus we get (2.12).

Now let $K$ be as in (2.11). We can apply the orbifold method since (2.12) holds. The question is whether the connection coming out of the orbifold is flat or not. In our case, the connection is flat iff (see [2]):

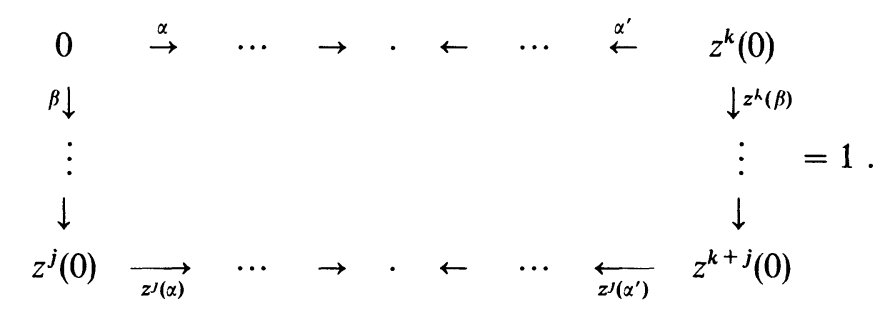

Here $z \in Z, Z \leqq Z(G), k, j$ are integers. The length of the string $\alpha$ is equal to that of $\alpha^{\prime}$. Let's denote the left-hand side of $(2.16)$ by $\mu\left(z^{k}, z^{j}\right)$.

We will find the necessary and sufficient condition of (2.16). First we prove the following lemma:

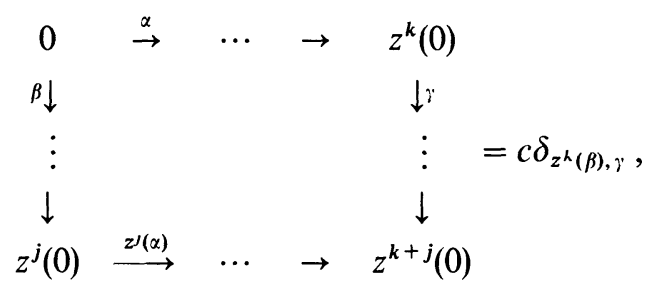

where $c$ is a number whose absolute value is 1 .

Proof. By using our convention for the connection as of (2.7), up to nonzero numbers depending only on the boundary terms, the left-hand side of the above 
expression is equal to the following diagrams: (The partition function associated to the diagram is defined as in Sect. one.)

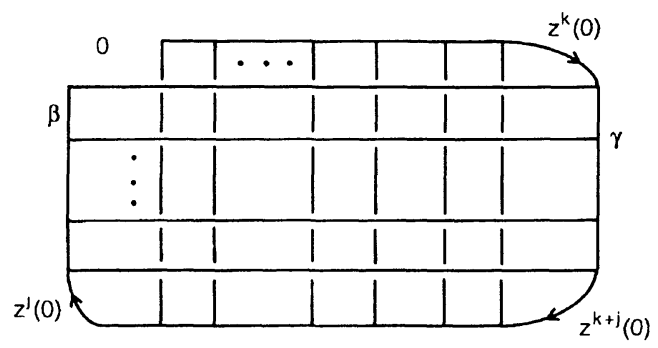

The reason is because the boundary terms of the above picture are simply the product of special fusion matrix elements which are nonzero by (1.3), (1.8), (1.9) and (1.10). By using the YBE (1.4) and braiding-fusing relation (1.5) of Sect. one, we get:

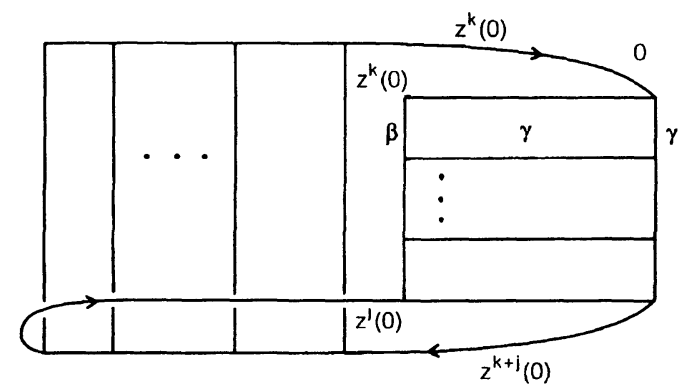

It's easy to see from the picture that one can get a nonzero number only if $\left(z^{k}(0), \beta ; \gamma\right)$ is admissible (see (c) of Sect. one). By $(2.10), z^{k}(0) \times \beta=z^{k}(\beta)$. Hence it is nonzero only if $z^{k}(\beta)=\gamma$, otherwise it's 0 . So:

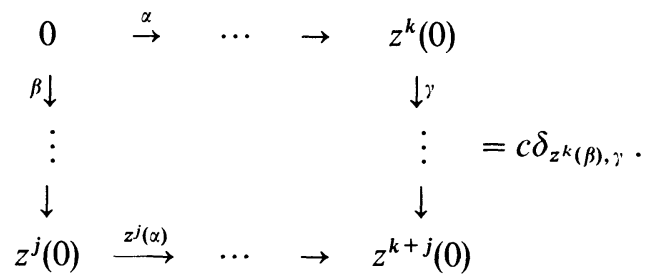

In the same way

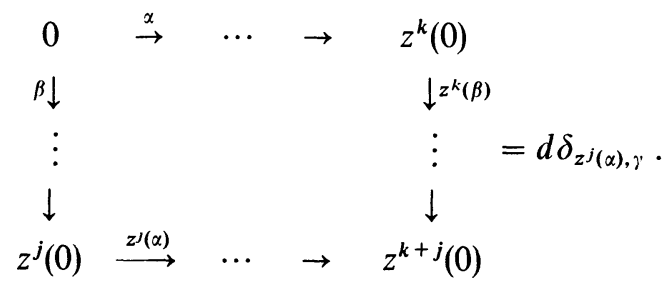


Hence

$$
\begin{array}{ccccccccc}
0 & \stackrel{\alpha}{\rightarrow} & \cdots & \rightarrow & z^{k}(0) & \leftarrow & \cdots & \stackrel{\alpha}{\leftarrow} & 0 \\
\beta \downarrow & & & & & & & & \downarrow^{\beta} \\
|c|^{2}=\begin{array}{ccccccc}
\downarrow \\
\vdots
\end{array} & & & & & & & & \vdots \\
z^{j}(0) & \underset{z^{\prime}(\alpha)}{\longrightarrow} & z^{k+j}(0) & \rightarrow & z^{k+j}(0) & \leftarrow & \cdots & \stackrel{z^{\prime}(\alpha)}{ } & z^{j}(0)
\end{array} .
$$

It follows that $|c|^{2}=1$ by the flatness of the original connection as in [1 and 2]. Next we show $\mu\left(z^{k}, z^{j}\right)$ is independent of $\alpha$ and $\alpha^{\prime}$. By renomalization rule,

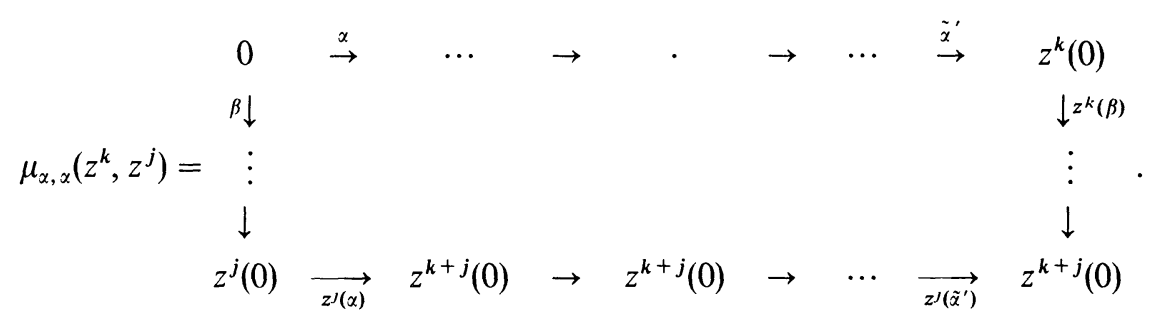

Hence:

$$
\begin{aligned}
& \left|\mu_{\alpha, \alpha^{\prime}}\left(z^{k}, z^{j}\right)-\mu_{\gamma, \gamma^{\prime}}\left(z^{k}, z^{j}\right)\right|^{2} \\
& \begin{array}{rlllllllll}
0 & \rightarrow & \cdots & \rightarrow & z^{k}(0) & \rightarrow & \cdots & \rightarrow & 0 \\
\beta \downarrow & & & & & & & & \downarrow^{\beta} \\
\vdots & \vdots & & & & & & & & \vdots \\
\downarrow & & & & & & & & \\
z^{j}(0) & \rightarrow & \cdots & \rightarrow & z^{k}(0) & \rightarrow & \cdots & \rightarrow & z^{j}(0)
\end{array},
\end{aligned}
$$

where the last step follows from Lemma 2.17.

So in order to prove the lemma, we need to show:

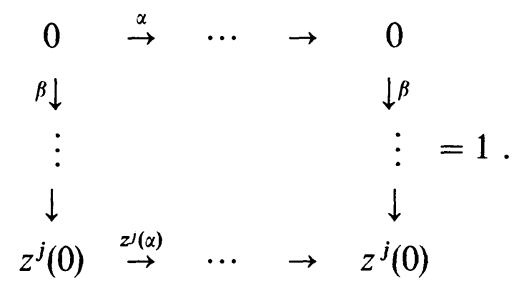

Let us use our notation for the connection to write (2.19). 
We have:

$\chi_{\phi}^{\frac{|\alpha|}{4}}$

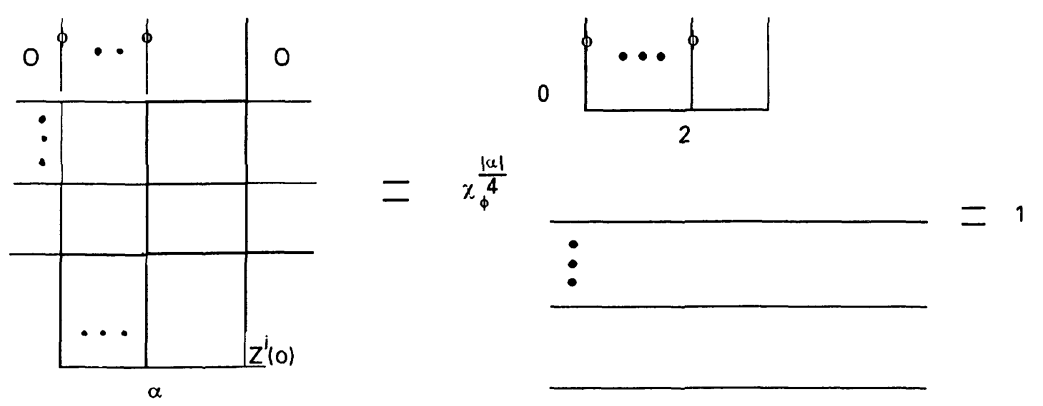

The above identity follows obviously from the topological invariance. This finishes the proof of (2.19).

Lemma 2.20. For fixed $z^{j}, \mu\left(\cdot, z^{j}\right)$ is a homomorphism from the cyclic subgroup generated by $z$ of $Z$ to the unit circle.

Proof. For example:

$$
\begin{aligned}
& \begin{array}{ccccc}
0 & \stackrel{\alpha}{\rightarrow} & \cdots & \rightarrow & z^{k}(0) \\
\beta \downarrow & & & & \downarrow z^{k(\beta)} \\
= & & & & \vdots \\
\downarrow & & & & \downarrow \\
z^{j}(0) \stackrel{z^{\prime}(\alpha)}{\longrightarrow} & z^{j+k}(0) \stackrel{z^{j+k}(\alpha)}{\longrightarrow} & z^{2 k+j}(0)
\end{array}
\end{aligned}
$$

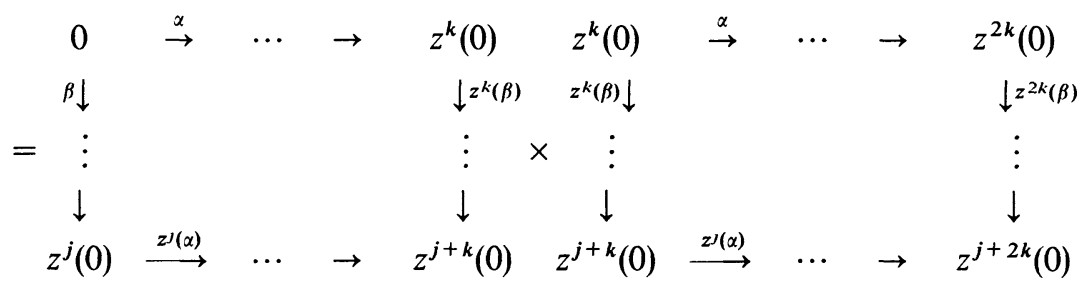

$$
\begin{aligned}
& =\mu\left(z^{k}, z^{j}\right) \mu\left(z^{k}, z^{j}\right) .
\end{aligned}
$$

Here the first identity follows from the independence of $\mu(\cdot, \cdot)$ on the paths, and the second follows from Lemma 2.17 and (2.12).

By Lemma 2.20 and 2.1 to 2.6 , where it's listed that $Z$ may be a cyclic group or $Z_{2} \times Z_{2}$, it's easy to see that $\mu\left(z^{k}, z^{j}\right)=1 \forall k, j$ iff $\mu\left(z^{k}, z^{-k}\right)=1 \forall k$.

For example, if $Z=Z_{t}, t \geqq 2$, choose $z=z_{0}$ to be the generator. Then $\mu\left(z^{k}, z^{j}\right)=\left(\mu\left(z^{0}, z^{0}\right)\right)^{k j}$ by $(2.20)$. Hence $(2.21)$ holds. The case when $Z=Z_{2} \times Z_{2}$ is similar.

Let $h_{z^{-1}}(0)$ be the conformal dimension of the weight $z^{-1}(0)$.

We have:

Lemma 2.21: $\mu\left(z, z^{-1}\right)=\exp \left( \pm 2 \pi i h_{z-1}(0)\right)$. 
Proof. Since $\mu\left(z . z^{-1}\right)$ is independent of the path, one may set:

$$
\mu\left(z, z^{-1}\right)=\begin{array}{ccccc}
0 & \stackrel{\alpha}{\rightarrow} & \cdots & \rightarrow & z(0) \\
\beta \downarrow & & & & \downarrow \tilde{\alpha} \\
\vdots & & & & \vdots \\
\downarrow & & & & \downarrow \\
z^{-1}(0) & \stackrel{\tilde{\beta}}{\rightarrow} & \cdots & \rightarrow & (0)
\end{array}
$$

Here $\beta, \tilde{\beta}$ are paths on $h$ and $\alpha, \tilde{\alpha}$ are paths on $g$.

By using (4.15) of [4] (our $\chi_{\phi}$ is $F_{\phi}$ in [4]):

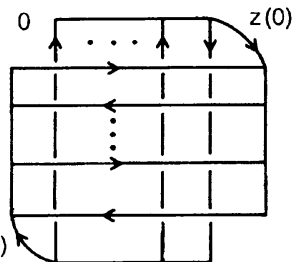

$$
\begin{aligned}
& =\chi_{\phi}^{-(2|\beta|+2|\alpha|) / 4} \times \\
& \begin{array}{|c|c|}
\hline \cdots & z(0) \\
\hline \vdots \\
\hline \cdots \\
\hline
\end{array} \\
& =\chi_{\phi}^{-(2|\beta|+2|\alpha|) / 4} \times \\
& \frac{z(0)}{\vdots} \\
& \mu\left(z, z^{-1}\right)=\chi_{\phi}^{-(2|\beta|+2|\alpha|) / 4} \times \\
& z^{-1}(0) \\
& =\chi_{\phi}^{-(2|\beta|+2|\alpha|) / 4} \times \exp \left( \pm 2 \pi i h_{z-1(0)}\right) \cdots \\
& =\exp \left( \pm 2 \pi i h_{z-1(0)}\right) \chi_{\phi}^{-(2|\beta|+2|\alpha|) / 4} \times \chi_{\phi}^{(2|\beta|+2|\alpha|) / 4} \\
& =\exp \left( \pm 2 \pi i h_{z-1(0)}\right) \text {, }
\end{aligned}
$$
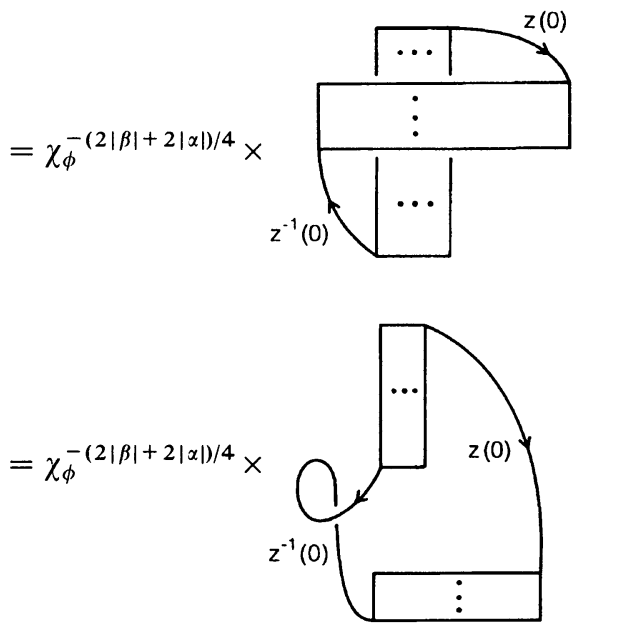

where the fourth identity follows from (1.6).

By (2.16), (2.20) and (2.21), we have proved the following theorem:

Theorem. Let $K$ be as in (2.11). Then the connection of the orbifold is flat iff $h_{z(0)} \in \mathbb{Z} \forall z \in Z$. 
Remark. Let $0_{Z}$ be the set of fundamental weights of $G$ associated to $Z$, let $M$ be the least number such that $1 / 2 M\left(\theta_{z}, 0_{z}\right) \in \mathbb{Z} \forall z \in Z$. Then $h_{z(0)} \in \mathbb{Z} \forall z \in Z$ is equivalent to $M \mid K$. This is noted in [8], where it's found that a Chern-Simons gauge theory based on $G / Z$ exists at level $K$ iff $M \mid K$.

\section{Appendix}

We include here an appendix on the part of the general theory of subfactors for the convenience of the reader. For more details, the reader is encouraged to consult the excellent expositions [11 and 3].

Let $N \subset M$ be type $\Pi_{1}$ factors. V. Jones determined the possible value of the index for a subfactor of a $\Pi_{1}$ factor and constructed a series of subfactors with indices $\left(4 \cos ^{2}\left(\frac{\pi}{n}\right)\right) \cup[4, \infty](n \geqq 3)$. A subfactor $N \subset M$ is called irreducible if $N^{\prime} \cap M=C$. The problem of classifying irreducible subfactors of the hyperfinite $\Pi_{1}$ factor is a big open question in operator algebra.

Starting with an inclusion of hyperfinite $\Pi_{1}$ factors with index $[M: N] \leqq \infty$, one can construct the associated Jones tower of factors $N \subset M \subset$ $M_{1} \subset M_{2} \subset \ldots$, where $M_{i+1}$ is the factor obtained from the Jones basic construction for $M_{i-1} \subset M_{1}$ (see [11]). The centralizer algebras $\left(M_{i}^{\prime} \cap M_{j}\right)_{i \leqq j}$ are finite dimensional $C^{\star}$ algebras. The following sequences of inclusions of algebras:

$$
\begin{gathered}
M^{\prime} \cap M_{k} \subset M^{\prime} \cap M_{k+1} \subset \ldots \\
\cup \quad \cup \\
M^{\prime} \cap M_{k} \subset M^{\prime} \cap M_{k+1} \subset \ldots
\end{gathered}
$$

is an invariant for the inclusion $N \subset M$, called paragroup (or the coupling system in [3]). They satisfy certain symmetry conditions, forming what's called a commuting square (see [11]).

The paragroups the underlying set of a group is replaced by a graph, the group elements are substituted by strings on the graph and a geometrical connection stands for the composition law. The harmonic analysis is similar to the computation of the partition function in statistical mechanics.

Let $R$ be the Murray-Von Neuman hyperfinite $\Pi_{1}$ factor. An important class of subfactors of $R$ are the finite depth subfactors, the finite depth refers to the condition $\sup _{k} \operatorname{dim} Z\left(M^{\prime} \cap M_{k}\right) \leqq \infty$, where $Z\left(M^{\prime} \cap M_{k}\right)$ denotes the center of $M^{\prime} \cap M_{k}$. The finite depth subfactors of $R$ are classified by the paragroup.

Oceanu axiomatized the paragroup for subfactors $N \subset M$ with finite depth. It consists of the following ingredients: Let $g_{1}, g_{2}, g_{3}, g_{4}$ be four finite graphs with the following properties:

(1) Each graph is bipartite, i.e., the vertices are divided into even and odd ones.

(2) Perron-Frobenius eigenvalues of the adjacent matrices coincide for $g_{2}$ and $g_{4}$ and for $g_{1}$ and $g_{3}$.

(3) Even vertices of $g_{2}$ and $g_{1}$ coincide, odd vertices of $g_{2}$ and $g_{3}$ coincide vertices of $g_{3}$ and $g_{4}$ coincide, and odd vertices of $g_{4}$ and $g_{1}$ coincide. 
Take a diagram:

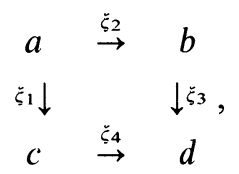

where $\xi_{j}$ 's satisfy one of the following: $\xi_{1} \in g_{1}, \xi_{2} \in g_{2}, \xi_{2} \in g_{3}, \xi_{4} \in g_{4}$,

or $\xi_{1} \in g_{3}, \xi_{2} \in g_{2}, \xi_{2} \in g_{1}, \xi_{4} \in g_{4}$,

or $\xi_{1} \in g_{1}, \xi_{2} \in g_{4}, \xi_{2} \in g_{3}, \xi_{4} \in g_{4}$,

or $\xi_{1} \in g_{3}, \xi_{2} \in g_{4}, \xi_{2} \in g_{1}, \xi_{4} \in g_{2}$,

and $a=s\left(\xi_{2}\right), b=r\left(\xi_{2}\right)=s\left(\xi_{3}\right), c=r\left(\xi_{1}\right)=s\left(\xi_{4}\right), d=r\left(\xi_{3}\right)=r\left(\xi_{4}\right)\left(\right.$ Here $s\left(\xi_{j}\right)$ and $r\left(\xi_{j}\right)$ mean the starting point and the ending point of the edge $\xi_{j}$.) Such a diagram is called a cell. A connection is an assignment of a complex number to each cell, and we write:

$$
W\left(\begin{array}{ccc}
a & \stackrel{\check{\zeta}_{2}}{\rightarrow} & b \\
\check{\zeta}_{1} \downarrow & & \downarrow \\
c & \stackrel{\check{\zeta}_{3}}{\rightarrow} & d
\end{array}\right) \in \mathbb{C}
$$

In the string algebra construction which is based on the four finite graphs $g_{1}, g_{2}, g_{3}, g_{4}$, the connection is used to embed a small string algebra naturally into a big one. The resulting sequences of inclusions of algebras are supposed to be the inclusions of algebras coming from the subfactors at the beginning of this appendix, so they form commuting squares, hence we have some restrictions on the connections. For example, we require the following renormalization rule:

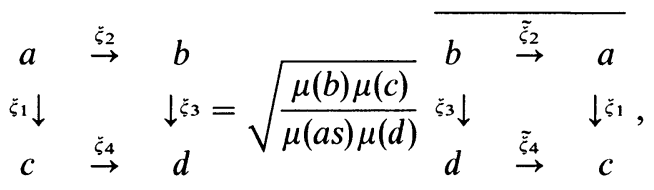

where $\tilde{\xi}_{j}$ means the edge with its orientation reversed and $\mu(\cdot)$ denotes an entry of the Perron-Frobenius eigenvector of the adjacent matrix of each graph. We also require the biunitarity condition:

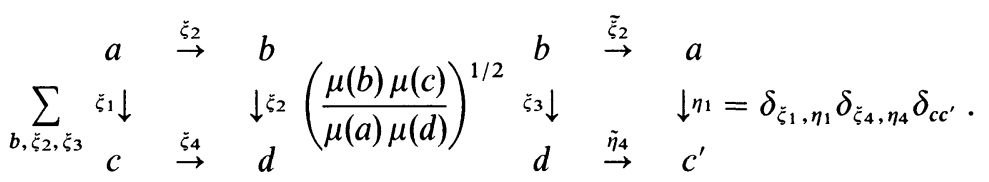

The above conditions are local conditions. There's an important global condition, called flatness condition, which will guarantee that the graphs (with connections on it) we used to construct subfactors will be the principal graph (and its dual) of the subfactors. To describe this flatness condition, choose two distinguished points $\otimes$ among the even vertices of $g_{1}$ and $g_{3}$ respectively. The connection is flat iff it satisfies the following condition for the double string algebra sequences and 
that with $g_{1}$ and $g_{3}$ interchanged:

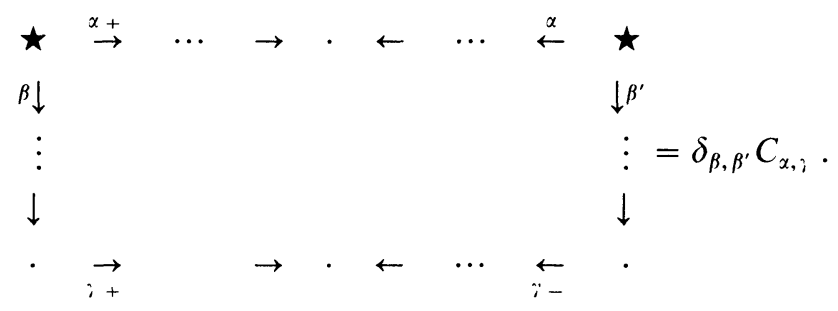

Here $C_{\alpha, \gamma}$, depends only on $\alpha, \gamma$ which are strings on $g_{1}$ and $g_{3}$ (see [1]).

It has been noticed for some time that the conditions on the connections, namely the symmetry (the renormalization rules), the biunitarity and the flatness conditions and very similar to various conditions on Boltzmann weights of restricted lattice in RCFT satisfy all the axioms of the paragroup (or coupling system). For e.g., using our notation (2.7) for the connection (and omitting the spin variables as usual), the biunitarity condition follows from:

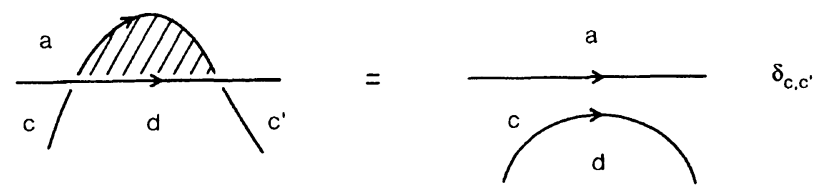

which is evident topologically.

We end up this appendix with the following correspondences between the theory of finite depth subfactors and that of RCFT:

Irreducible subfactors of finite depth RCFT

Finite depth condition ....... Rationality

Connections ....... Suitably normalized braiding matrices

Principal graphs and its dual ....... Fusion graphs of primary field and its dual

Flatness ....... Topological invariance (e.g., YBE, braiding-fusion relations).

The symmetries in subfactors coming from the commuting square condition, but in RCFT they come from the polynomial equations of Moore and Seiberg. Of course, one needs to take the above correspondences with a grain of salt. For e.g., the principal graphs and its dual are always isomorphic for those subfactors coming from RCFT. But we know that there are interesting subfactors (exclude those coming from finite group action whose structures are well-known) with finite depth with principal graph (as an unlabelled graph) different from its dual. It's an interesting question to seen if one can find a corresponding construction in RCFT.

Acknowledgements. My advisor V. Jones patiently introduced me to the subject of subfactors. I'm greatful to Y. Kawahigashi for his explainations of [1] and [2] before their publications and his suggestions. I would like to thank N.Yu.Reshetikhin and D. Bisch for helpful discussions.

\section{References}

1. Kawahigashi, Y., Evans, D.E.: Orbifold subfactors from Hecke algebra (to appear)

2. Kawahigashi, Y.: On flatness of Oceanu's connections on the Dykin diagrams and classifications of subfactors. (To appear) 
3. Ocneanu, A.: Quantum symmetry, differential geometry of finite graphs and classification of subfactor. University of Tokyo Seminary Notes 45 (recorded by Y. Kawahigashi), 1991

4. De boer, J., Goeree, J.: $\Pi_{1}$ factors and Rational conformal field theory. Commun. Math. Phys. 131, 267-304 (1991)

5. Moore, G., Seiberg, N.: Commun. Math. Phys. 123, 77 (1989)

6. Pasquier, L.: Commun. Math. Phys. 116, 564 (88)

7. Li, M.: Commun. Math. Phys. 137, 473-525 (1991)

8. Witten, E., Dijkgraf, K.: Commun. Math. Physics 127, 10 (1990)

9. Walton,: Fusion rule of Wees-Zumino-Witten Models. Nucl. Phys. B 340, 777-789

10. Jones, V.: Baxterization. Int. J. Mod. Phys. A5 (90), 456

11. Goodman, F.M., de la Harpe, P., Jones, V.: Towers of algebras and coxeter graphs MSRI publications, no 14,90

12. Witten, E.: Gauge theories and integrable lattice models. Nuclear Physics, to appear

13. Frohlich, J., King, C.: Int. Journal of Modern Physics A4, 5321-5342 (89)

Communicated by N. Yu. Reshetikhin 
